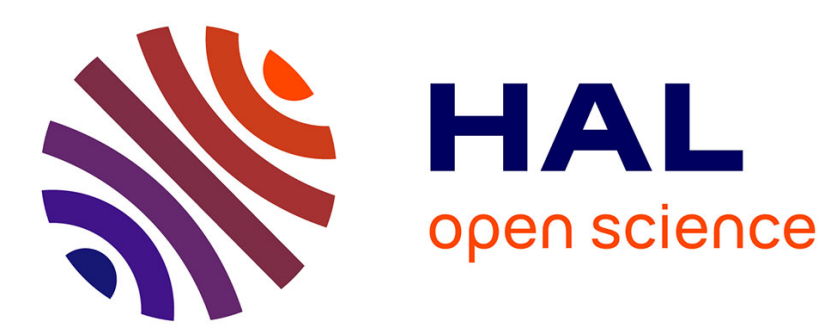

\title{
Thermocourants de dépolarisation et distribution de temps de relaxation diélectrique
}

\author{
R.J. Heitz, H. Szwarc
}

\section{To cite this version:}

R.J. Heitz, H. Szwarc. Thermocourants de dépolarisation et distribution de temps de relaxation diélectrique. Revue de Physique Appliquée, 1980, 15 (3), pp.687-696. 10.1051/rphysap:01980001503068700 . jpa-00244776

\section{HAL Id: jpa-00244776 https://hal.science/jpa-00244776}

Submitted on 1 Jan 1980

HAL is a multi-disciplinary open access archive for the deposit and dissemination of scientific research documents, whether they are published or not. The documents may come from teaching and research institutions in France or abroad, or from public or private research centers.
L'archive ouverte pluridisciplinaire HAL, est destinée au dépôt et à la diffusion de documents scientifiques de niveau recherche, publiés ou non, émanant des établissements d'enseignement et de recherche français ou étrangers, des laboratoires publics ou privés. 


\title{
Thermocourants de dépolarisation et distribution de temps de relaxation diélectrique
}

\author{
R. J. Heitz et H. Szwarc \\ Laboratoire de Chimie-Physique des Matériaux Amorphes $\left({ }^{*}\right)$, Université de Paris-Sud, 91405 Orsay, France
}

(Reçu le 26 septembre 1979, révisé le 5 décembre 1979, accepté le 6 décembre 1979)

\begin{abstract}
Résumé. - Nous présentons une méthode numérique d'exploitation des mesures de thermocourants de dépolarisation en termes de fonction de distribution des temps de relaxation diélectrique; cette méthode, appliquée à un halogénure d'alkyle, donne un excellent accord avec des mesures diélectriques traditionnelles au-dessus de la température $T_{\mathrm{g}}$ de transition vitreuse. Elle nous a permis de décrire le domaine de relaxation principal, ou domaine $\alpha$, de part et d'autre de $T_{\mathrm{g}}$, ainsi qu'un domaine secondaire $\beta$ culminant $\sim 30 \mathrm{~K}$ au-dessous de $T_{\mathrm{g}}$ dans nos expériences. Elle révèle aussi une inflexion dans la courbe $\operatorname{Ln} \tau_{M}=\varphi(1 / T)\left(\tau_{M}=\right.$ temps de relaxation au maximum de l'absorption diélectrique) aux alentours de $T_{\mathrm{g}}$; cette inflexion pourrait être l'indice que $\tau_{\mathrm{M}}$ tend vers une valeur finie à basse température.
\end{abstract}

\begin{abstract}
We present a computational method describing depolarization thermo-current measurements in terms of dielectric relaxation time distribution functions. This method has been applied to an amorphous alkyl halide; its results are in excellent agreement with those recorded through conventional dielectric mesurements at temperatures higher than $T_{\mathrm{g}}$, the glass transition temperature. We were able to give distribution functions for the main relaxation domain around $T_{\mathrm{g}}$ and for a secondary $\beta$ domain which reaches a maximum $30 \mathrm{~K}$ lower than $T_{\mathrm{g}}$ in our experiments. We also observed that the curve $\operatorname{Ln} \tau_{\mathrm{M}}=\varphi(1 / T)$ (where $\tau_{\mathrm{M}}$ is the relaxation time at maximum dielectric absorption) presents an inflexion point near $T_{\mathbf{g}}$. We think this inflexion point to be a clue for $\tau_{M}$ reaching a finite value at low temperatures.
\end{abstract}

1. Introduction. - La mesure des thermocourants de dépolarisation (TCD) est une méthode d'étude des interactions moléculaires en phase dense. Cette méthode, introduite en 1963 par Bucci et al. [1, 2], consiste à polariser le milieu moléculaire étudié par un champ électrique $E$ non saturant, puis à geler la configuration des molécules soumises au champ $E$ par refroidissement, ensuite à supprimer le champ et à réchauffer lentement afin d'enregistrer le thermocourant de dépolarisation (TCD) en fonction de la température. Cette méthode s'apparente à la mesure de l'absorption diélectrique à fréquence fixe en fonction de la température. Toutefois l'absorption diélectrique est mesurée le plus souvent à température fixe en fonction de la fréquence, ce qui facilite l'interprétation des résultats.

Mais les mesures diélectriques proprement dites deviennent impossibles à des fréquences inférieures à $10^{-4}-10^{-5} \mathrm{~Hz}$. Or, après le travail de Denney [3], on peut admettre que la viscosité et le temps de relaxation

(*) Partie du laboratoire associé au C.N.R.S. n" 75. diélectrique au maximum de l'absorption correspondante restent proportionnels dans un liquide surfondu quand la température décroît jusqu'à la transition vitreuse. D'autre part, depuis les travaux de Tammann [4], il est courant de prendre comme définition opérationnelle de la température de transition vitreuse $T_{\mathrm{g}}$, la température à laquelle la viscosité du milieu amorphe est égale à $10^{13} \mathrm{P}$. Il s'ensuit que l'on peut considérer, dans le même cadre, que $T_{\mathrm{g}}$ correspond à la température où la fréquence au maximum de l'absorption est égale à $10^{-5} \mathrm{~Hz}$, si bien que l'étude de l'absorption diélectrique principale dans les verres ne peut être réalisée par les moyens conventionnels.

Or les TCD permettent d'accéder à des fréquences bien inférieures à $10^{-5} \mathrm{~Hz}$ et sont donc naturellement destinés à prolonger les mesures diélectriques dans ce domaine de fréquence.

Le principal inconvénient des TCD consiste en ce que la température varie au cours de l'expérience, ce qui rend très difficile l'exploitation des résultats. Le but du présent travail est donc de présenter une méthode de calcul capable de rendre compte de pics de TCD résultant de distributions, symétriques ou 
asymétriques, de temps de relaxation diélectrique, dans les conditions expérimentales les plus variées.

2. Méthode d'étude des distributions. - Quelques auteurs ont déjà tenté d'étudier les distributions de temps de relaxation par les TCD et nous pensons utile de résumer leurs travaux. Deux méthodes principales ont été proposées : la première consiste à simuler sur ordinateur les pics de TCD, puis à comparer un pic expérimental avec des pics simulés jusqu'à obtenir leur superposition, l'autre méthode consiste à décomposer expérimentalement un pic distribué en plusieurs pics, par polarisation à une température $T_{\mathrm{p}}$ suivie d'une dépolarisation à une température inférieure de un degré par exemple : par réchauffement lent un pic de TCD est alors enregistré. En faisant varier $T_{\mathrm{p}}$ de degré en degré, le pic distribué est remplacé par une somme de pics élémentaires, dont on admet qu'ils sont non distribués.

Van Turnhout $[5,6]$ a utilisé la première méthode et uniquement pour simuler des pics de distribution symétrique, de type gaussien. Le courant $I(T)$ du pic de TCD est obtenu par sommation d'intégrales sur un même petit intervalle de température, à partir de la plus basse température. Malheureusement l'utilisation de relations approchées, comme celle de Schwarzl et Staverman [7] conduit à des pics de TCD simulés qui s'éloignent fortement de la courbe mathématiquement rigoureuse vers les hautes températures, et ne permet de simuler que des polarisations et dépolarisations à températures fixes ou variant linéairement. La deuxième méthode (décomposition expérimentale d'un pic distribué de TCD en pics non distribués) a été proposée dans plusieurs articles récents pour l'étude des pics distribués de TCD apparaissant dans les polymères au voisinage de la transition vitreuse : $T_{\mathrm{g}}(\mathrm{pic} \alpha)$ et en dessous de $T_{\mathrm{g}}(\mathrm{pic} \beta)$ [8 à 11]. Cette méthode s'applique lorsqu'il est possible de polariser un ensemble de dipôles de même temps de relaxation $\tau$, avec un même facteur préexponentiel $\tau_{0}$ et une même énergie d'activation $E_{0}$, que l'on peut représenter par une équation de type Arrhenius :

$$
\tau=\tau_{0} \exp \left(\frac{E_{0}}{k T}\right)
$$

où $k$ est la constante de Boltzmann et $T$ la température.

Cette dernière condition n'est pas vérifiée pour les pics de TCD distribués, où $\tau_{0}$ peut prendre toutes les valeurs $\tau^{\prime}$ de zéro à l'infini avec un poids $G\left(\tau^{\prime}\right)$. En effet si l'on polarise à la température fixe $T_{1}$ pendant un temps $t_{1}$, on doit multiplier la fonction de distribution initiale $G\left(\tau^{\prime}\right)$ par une nouvelle fonctionpoids $H_{1}\left(\tau^{\prime}\right)$ :

$$
H_{1}\left(\tau^{\prime}\right)=1-\exp \left[-\frac{t_{1}}{\tau^{\prime}} \exp \left(-\frac{E_{0}}{k T_{1}}\right)\right]
$$

qui rend compte du fait que la polarisation n'est pas complète. De même en dépolarisant à la température fixe $T_{2}$ pendant un temps $t_{2}$, on doit faire intervenir une deuxième fonction poids :

$$
H_{2}\left(\tau^{\prime}\right)=\exp \left[-\frac{t_{2}}{\tau^{\prime}} \exp \left(-\frac{E_{0}}{k T_{2}}\right)\right]
$$

facteur multiplicatif supplémentaire de $G\left(\tau^{\prime}\right)$. La deuxième méthode conduit donc à une décomposition expérimentale en pics élémentaires distribués selon la fonction poids : $H_{1}\left(\tau^{\prime}\right) \times H_{2}\left(\tau^{\prime}\right) \times G\left(\tau^{\prime}\right)$, ce qui ne peut se réduire à un pic non distribué puisque $G\left(\tau^{\prime}\right)$ correspond déjà à la fonction poids d'un pic distribué et que $H_{1}\left(\tau^{\prime}\right)$ comme $H_{2}\left(\tau^{\prime}\right)$ ont des valeurs non nulles pour une infinité de valeurs de $\tau^{\prime}$ pour tout couple de valeurs $t_{1}$ et $T_{1}$ ou $t_{2}$ et $T_{2}$ non nulles.

Pour l'étude des pics $\alpha$ et $\beta[12,13]$ aux températures voisines ou inférieures à celle de la transition vitreuse, seule la méthode utilisée par Van Turnhout peut convenir. C'est donc une méthode de ce type que nous présentons dans cet article, en utilisant le calcul numérique pour trouver la solution des équations intégro-différentielles donnant le TCD et la polarisation : $P$, en fonction de la température. En évitant d'utiliser des relations algébriques approchées par des développements limités dont la convergence est trop lente, nous obtenons une précision nettement supérieure et pouvons simuler des polarisations et dépolarisations à température variant selon une loi quelconque.

3. Equation du courant de dépolarisation. - 3.1 CAs de PICS De TCD de Debye. - Dans une expérience de TCD, l'échantillon du corps à étudier constitue le diélectrique d'un condensateur et est soumis à un champ électrique à une température suffisamment élevée : $T_{\mathrm{p}}$, pour permettre l'orientation

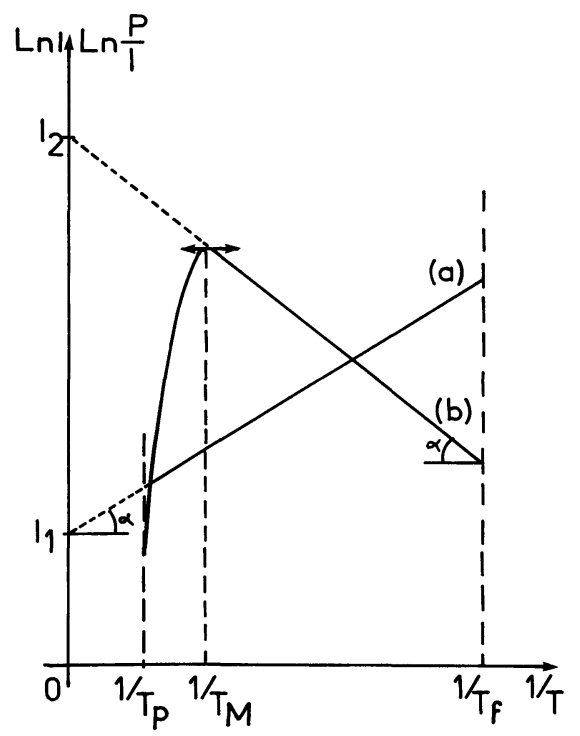

Fig. 1. - Courbe (a) : $\operatorname{Ln}(P / I)$ et courbe (b) $: \operatorname{Ln}(I)$ en fonction de $1 / T$ pour les TCD. $\operatorname{Ln}\left(I_{1}\right)=\tau_{0} ; \operatorname{Ln}\left(I_{2}\right)=P_{0} / \tau_{0} ; \operatorname{tg} \alpha=E / k$.

[Curve (a) : $\operatorname{Ln}(P / I)$ and curve (b) : Ln $(I)$ versus $1 / T$ for DTC. $\operatorname{Ln}\left(I_{1}\right)=\tau_{0} ; \operatorname{Ln}\left(I_{2}\right)=P_{0} / \tau_{0} ; \operatorname{tg} \alpha=E / k$.] 
des dipôles; puis l'échantillon est fortement refroidi jusqu'à $T_{\mathrm{f}}$ où les mouvements des dipôles sont bloqués; le champ électrique est alors supprimé. Ensuite un réchauffement lent, de vitesse $b(T)\left(\mathrm{en} \mathrm{Ks}^{-1}\right.$ ) fait apparaître un thermocourant de dépolarisation, $I(T)$, aux bornes du condensateur. Si l'équation (1) est vérifiée, c'est-à-dire si $E_{0}$ et $\tau_{0}$ sont constants (pic de Debye) :

$$
\begin{aligned}
I(T)= & \frac{P(T)}{\tau(T)}=\frac{P_{0}}{\tau_{0}} \exp \left(-\frac{E_{0}}{k T}\right) \times \\
& \times \exp \left[-\int_{T_{\mathrm{r}}}^{T} \frac{1}{b(T) \tau_{0}} \exp \left(-\frac{E_{0}}{k S}\right) \mathrm{d} S\right]
\end{aligned}
$$

où $P$ est la polarisation de l'échantillon $\left(P=P_{0}\right.$ pour $\left.T=T_{\mathrm{f}}\right)$. Ln $I$ et $\operatorname{Ln}(P / I)$ sont habituellement tracés en fonction de $1 / T$ (Fig. 1), car la pente de la partie linéaire de ces courbes est égale à $\pm E / k$.

La dérivée de $I$ par rapport à $T$ est nulle pour $T=T_{\mathrm{M}}$; on obtient à partir de l'équation (4) :

$$
\tau_{0}=T_{\mathrm{M}}^{2} \frac{k}{b E_{0}} \exp \left(-\frac{E_{0}}{k T_{\mathrm{M}}}\right)
$$

d'où $\tau_{0}$.

3.2 CAS DE PICS DE TCD DISTRIBUÉs. - Pour un pic de Debye (Fig. 1) les deux courbes précédentes permettent de déterminer aisément $E_{0}$ et $\tau_{0}$ (éqs (1), (4) et (5)). Dans le cas d'un pic distribué (Figs. 2, 4 et 6) les mêmes courbes n'ont plus de partie rectiligne, sauf pour les températures très inférieures à celle du sommet du pic de courant : $T_{\mathrm{M}}$; pour $T \ll T_{\mathrm{M}}$ la pente de la courbe $\operatorname{Ln} I=\varphi(1 / T)$ est égale à $-c E_{0} / k$. Le coefficient $c$ est égal à l'unité pour une distribution gaussienne et supérieur à l'unité pour les autres distributions; il a déjà été calculé dans le cas de certaines distributions symétriques $[5,6]$. De même, la pente de la courbe $\operatorname{Ln}(P / I)=\varphi(1 / T)$ est égale à $+c E_{0} / k$ pour $T \ll T_{\mathrm{M}}$.

Ce procédé de détermination de $E_{0}$ est peu précis, car la mesure de la pente des courbes se fait dans une région de courants faibles, où ces courants sont mesurés avec une précision médiocre. Nous croyons préférable de mesurer la pente de la courbe

$$
\operatorname{Ln}(P / I)=\varphi(1 / T)
$$

aux environs de la température $T_{\mathrm{M}}$ du maximum de courant, pente égale à :

$$
\operatorname{tg} \theta_{\mathrm{M}}=A E_{0} / k
$$

$A$ est un coefficient semblable au coefficient $c$ précédent. Nous déterminerons le coefficient $A$ pour des distributions classiques de temps de relaxation, symétrique et asymétrique. Pour la détermination de $\tau_{c}$, temps de relaxation caractéristique d'une distribution (voir ci-dessous pour des exemples), nous déterminerons le coefficient $B$ de l'équation (5) modifiée :

$$
\tau_{\mathrm{c}}=\frac{T_{\mathrm{M}}^{2}}{B} \frac{k}{b E_{0}} \exp \left(-\frac{E_{0}}{k T_{\mathrm{M}}}\right)
$$

pour ces mêmes distributions classiques.

Ainsi $E_{0}$ et $\tau_{\mathrm{c}}$ seront déterminés par des mesures faites uniquement à $T_{\mathrm{M}}$ où le courant est maximum, donc avec une grande précision, lorsque la distribution est connue.

3.3 EQUATIONS CORRESPONDANT A UNE DISTRIBUTION SUR $\tau_{0}$. - Dans le cas d'une distribution sur $\tau_{0}$, le terme $\tau_{0}$ de l'équation (1) peut prendre toute valeur nulle ou positive, selon une fonction de distribution normalisée $G(\tau)$, telle que :

$$
\int_{0}^{\infty} G(\tau) \mathrm{d} \tau=1
$$

et, du fait de l'additivité des TCD, l'équation (4) devient :

$$
\begin{aligned}
I(T)= & \int_{0}^{\infty} G(\tau) \frac{P_{0}}{\tau} \exp \left(-\frac{E}{k T}\right) \times \\
& \times \exp \left[-\int_{T_{\mathrm{f}}}^{T} \frac{1}{b(T) \tau} \exp \left(-\frac{E_{0}}{k S}\right) \mathrm{d} S\right] \mathrm{d} \tau .
\end{aligned}
$$

Notons qu'une polarisation incomplète exige que l'on remplace dans l'équation (9) le terme $P_{0}$ par [5] :

$$
\begin{aligned}
P_{0}\{1-\exp & {\left[-\frac{t_{\mathrm{p}}}{\tau} \exp \left(-\frac{E_{0}}{k T_{\mathrm{p}}}\right)-\right.} \\
& \left.\left.-\int_{T_{\mathrm{p}}}^{T_{\mathrm{f}}} \frac{1}{b_{\mathrm{p}} \tau} \exp \left(-\frac{E_{0}}{k T}\right) \mathrm{d} T\right]\right\}
\end{aligned}
$$

où $b_{\mathrm{p}}$ est la vitesse de refroidissement sous champ électrique, $T_{\mathrm{p}}$ et $t_{\mathrm{p}}$ respectivement la température et le temps de polarisation.

Pour résoudre l'équation (9) nous fixons l'intervalle de variation de $T \in\left(T_{\mathrm{a}}, T_{\mathrm{b}}\right)$, avec le plus souvent $T_{\mathrm{a}}=T_{\mathrm{f}}$ et $\left(T_{\mathrm{M}}-T_{\mathrm{b}}\right) \sim 3\left(T_{\mathrm{a}}-T_{\mathrm{M}}\right)$. L'intervalle $\left(T_{\mathrm{a}}\right.$, $T_{\mathrm{b}}$ ) est alors divisé en 400 sous-intervalles et dans chaque sous-intervalle la formule d'intégration numérique de Gauss-Legendre à 12 pivots est appliquée pour le calcul de :

$$
\int_{T_{\mathrm{f}}}^{T} \frac{1}{b} \exp \left(-\frac{E_{0}}{k S}\right) \mathrm{d} S
$$

puis l'intervalle $\left(T_{\mathrm{a}}, T_{\mathrm{b}}\right)$ est divisé à nouveau, cette fois en 1200 sous-intervalles, et la formule d'intégration de Gauss-Legendre à 24 pivots est appliquée pour l'intégration sur $\tau$.

Cette méthode d'intégration numérique où les deux intégrales imbriquées sont calculées successivement, 
permet d'introduire des vitesses de réchauffement $b(T)$ variables avec la température, mais exige d'utiliser une fonction de distribution $G(\tau)$ indépendante de la température. Cette méthode permet également d'introduire des polarisations incomplètes en multipliant $P_{0}$ dans l'équation (9) par le facteur donné dans l'équation (9bis).

Nous déterminons ensuite la polarisation $P(T)$ par :

$$
P(T)=\int_{0}^{t} I(T) \mathrm{d} t=\int_{T_{\mathrm{f}}}^{T} I(T) \frac{\mathrm{d} T}{b(T)}
$$

où $b(T)=\mathrm{d} T / \mathrm{d} t$, en intégrant par la méthode des trapèzes sur l'intervalle $\left(T_{\mathrm{a}}, T_{\mathrm{b}}\right)$ divisé en 100 sousintervalles égaux.

Lorsque $T$ est suffisamment élevé pour que toute la polarisation de l'échantillon ait disparu, ce qui se produit généralement pour $T=T_{\mathrm{b}}, P\left(T_{\mathrm{b}}\right)$ devrait redonner $P_{0}$. Ce test global permet d'obtenir une estimation de l'erreur introduite par les intégrations numériques précédentes. Nous a vons toujours obtenu :

$$
\frac{P_{0}-P\left(T_{\mathrm{b}}\right)}{P_{0}}<10^{-4}
$$

avec un ordinateur conservant 9 chiffres décimaux significatifs en mémoire pour chaque nombre introduit.

4. Etude de distributions classiques de temps de relaxation - Les coefficients $A$ et $B$ des équations (6) et (7) ont été déterminés en simulant sur ordinateur les TCD correspondant à $E_{0}=0,15 \mathrm{eV}, \tau_{\mathrm{c}}=10^{-12} \mathrm{~s}$ et $b=0,1 \mathrm{~K} / \mathrm{s}$, pour des distributions standards [14] (Figs. 3, 5 et 7).

Cette façon de procéder n'est valable que si la forme des courbes $\operatorname{Ln} I$ et $\operatorname{Ln}(P / I)=\varphi(1 / T)$ est indépendante de $\tau_{\mathrm{c}}$ et $b$. Les auteurs qui mesurent la pente $c E_{0} / k$ pour $T \ll T_{\mathrm{M}}$ admettent implicitement cette propriété. En fait la forme des courbes dépend très légèrement du produit $b . \tau_{c}$, mais si peu qu'il est possible d'admettre une pente indépendante de $b . \tau_{c}$ avec une excellente approximation. Le seul cas où nous avons pu mettre en évidence une pente dépendant très légèrement du produit $b . \tau_{\mathrm{c}}$ est indiqué sur la figure 4 , pour une distribution de Cole et Cole avec $b . \tau_{\mathrm{c}}=10^{-13}$ (traits pleins) puis $10^{-18}$ (traits pointillés). Mais il n'apparaît pas de modification de la forme des courbes au voisinage de $T_{\mathrm{M}}$ où nous mesurons la pente (éq. (6)), ce qui justifie notre méthode.

4.1 DisTRIBUTIONS SYMÉTRIQUES. - Comme nous l'avons signalé précédemment Van Turnhout [5] a étudié des distributions symétriques, en faisant usage de formules approchées. Sa conclusion est que l'on ne peut pas distinguer entre les courbes obtenues pour diverses équations de distributions symétriques (celles de Gevers, Wagner, Cole et Cole, Fuoss et Kirkwood). Nous nous sommes limités à présenter deux distributions symétriques; en fait, étant donné la précision des mesures, une seule de ces distributions permettrait de représenter tout résultat expérimental correspondant à une distribution symétrique.

a) Distribution symétrique gaussienne. - L'expression de $G(\tau)$ dans l'équation (9) est [14] :

$$
G(\tau)=\frac{1}{\tau} \frac{\delta}{\sqrt{\pi}} \exp \left[-\left(\delta \operatorname{Ln} \frac{\tau}{\tau_{\mathrm{c}}}\right)^{2}\right]
$$

où $\delta$ donne l'amplitude de dispersion, nulle pour $\delta \rightarrow+\infty$, et où $\tau_{\mathrm{c}}$ est le temps de relaxation pour lequel la fonction $G(\tau)$ est maximale. La figure 2 donne un exemple des courbes obtenues pour une distribution gaussienne, comparées aux courbes pour un pic de Debye de même énergie. On constate l'identité des

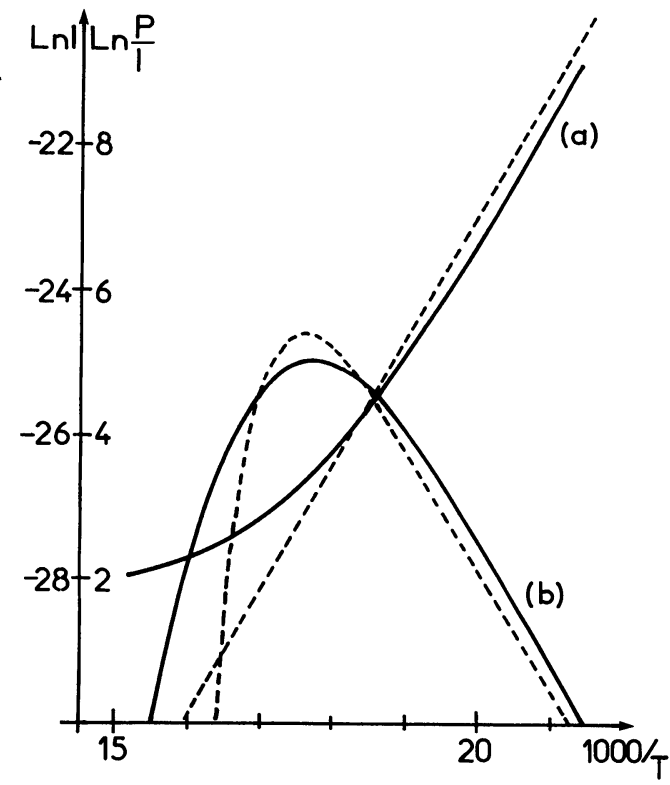

Fig. 2. - Courbe (a) : $\operatorname{Ln}(P, I)$ et courbe (b) : $\operatorname{Ln}(I)$ pour $P_{0}=10^{-9}, b=0,1 \mathrm{Ks}^{-1}, \tau_{0}=10^{-12} \mathrm{~s}$ et $E_{0}=0,15 \mathrm{eV}$, sans distribution en traits interrompus et pour une distribution gaussienne, $\delta=0,7$ et $\tau_{\mathrm{c}}=10^{-12}$ en traits continus.

[Curve (a) : $\operatorname{Ln}(P / I)$ and curve (b) : $\operatorname{Ln}(I)$ for $P_{0}=10^{-9}$, $b=0.1 \mathrm{Ks}^{-1}, \tau_{0}=10^{-12} \mathrm{~s}$ and $E_{0}=0.15 \mathrm{eV}$, for no distribution in dashed lines and for a Gaussian distribution, $\delta=0.7$ and $\tau_{\mathrm{c}}=10^{-12} \mathrm{~s}$ in continuous lines.]

pentes $\pm E_{0} / k$ pour $T \ll T_{\mathrm{M}}$ et $I<10^{-2} I_{\mathrm{M}}\left(I=I_{\mathrm{M}}\right.$ pour $T=T_{\mathrm{M}}$ ). Mais expérimentalement il est exceptionnel d'obtenir la partie du pic de TCD où $I<10^{-2} I_{\mathrm{M}}$. Souvent même la précision expérimentale n'est correcte que pour $I \geqslant 10^{-1} I_{M}$, de sorte qu'il n'est pas possible de comparer les pentes pour $I<10^{-2} I_{\mathrm{M}}$ et $T \ll T_{\mathrm{M}}$. Par contre notre méthode, mesurant la pente de $\operatorname{Ln}(P / I)$ pour $T_{\mathrm{M}}$ s'applique. Les coefficients $A(\delta)$ et $B(\delta)$, définis par les équations (6) et (7) sont donnés graphiquement sur la figure 3 . 


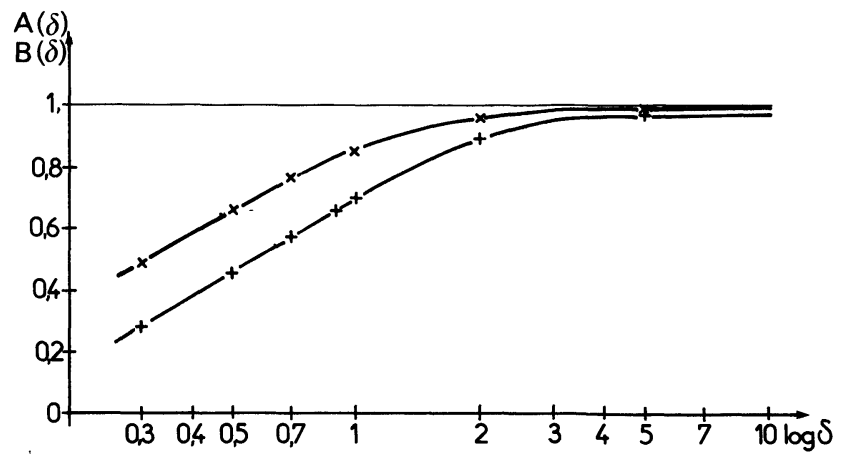

Fig. 3. $-+++: A(\delta)$ et $\times \times \times: B(\delta)$ pour une distribution gaussienne.

$[+++: A(\delta)$ and $\times \times \times: B(\delta)$ for a Gaussian distribution. $]$

b) Distribution symétrique de Cole et Cole.L'expression de $G(\tau)$ dans l'équation (9) devient [14, 15] :

$$
G(\tau)=\frac{1}{2 \pi \tau} \frac{\sin \alpha \pi}{\cosh \left(\alpha \operatorname{Ln} \frac{\tau}{\tau_{\mathrm{c}}}\right)+\cos (\alpha \pi)}
$$

avec $0<\alpha \leqslant 1$

où $\alpha$ donne l'amplitude de la dispersion, nulle pour $\alpha$ égal à l'unité, et $\tau_{\mathrm{c}}$ est le temps de relaxation pour lequel $G(\tau)$ est maximal. La figure 4 donne un exemple des courbes obtenues pour une distribution de Cole et Cole et la figure 5 donne graphiquement les coefficients $A(\alpha)$ et $B(\alpha)$ définis par les équations (6) et (7).

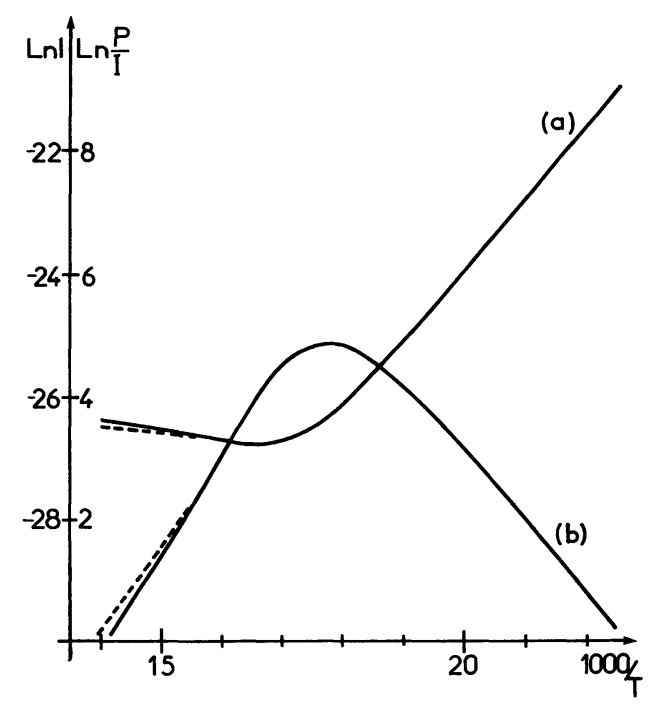

Fig. 4. - Courbe (a) $: \operatorname{Ln}(P / I)$ et courbe (b) $: \operatorname{Ln}(I)$ pour une distribution de Cole et Cole : $\alpha=0,7, \tau_{\mathrm{c}}=10^{-12} \mathrm{~s}, E_{0}=0,15 \mathrm{eV}$ et $b=0,1 \mathrm{Ks}^{-1}$. Les tirets montrent la modification des courbes pour $\tau_{\mathrm{c}}=10^{-17} \mathrm{~s}$ et $b=0,5 \mathrm{Ks}^{-1}$.

[Curve (a) : $\operatorname{Ln}(P / I)$ and curve (b) : $\operatorname{Ln}(I)$ for a Cole and Cole distribution : $\alpha=0.7, \tau_{\mathrm{c}}=10^{-12} \mathrm{~s}, E_{0}=0.15 \mathrm{eV}$ and $b=0.1 \mathrm{Ks}^{-1}$. The dashed lines indicate the shape alteration for $\tau_{\mathrm{c}}=10^{-17} \mathrm{~s}$ and $b=0.5 \mathrm{Ks}^{-1}$.]

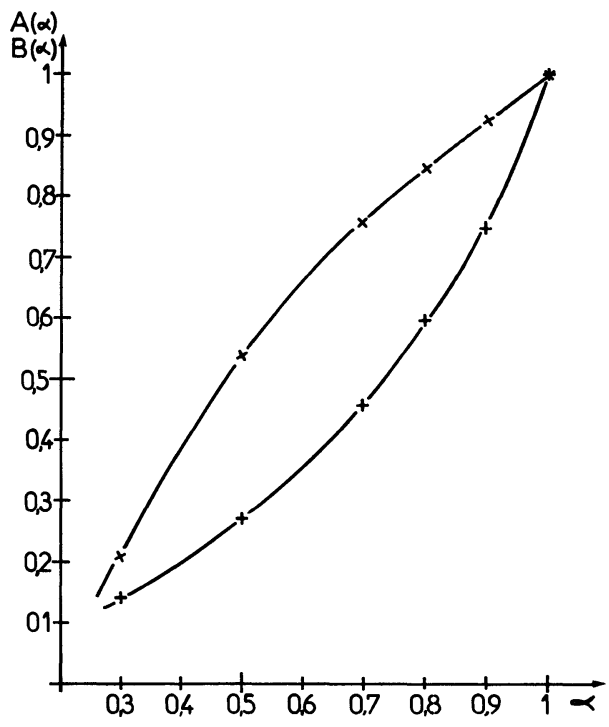

Fig. 5. $-+++: A(\alpha)$ et $\times \times \times: B(\alpha)$ pour une distribution de Cole et Cole.

$[+++: A(\alpha)$ and $\times \times \times: B(\alpha)$ for a Cole and Cole distribution. $]$

4.2 Distribution asymÉtriQue De DAVIDSON ET ColE. - L'expression de $G(\tau)$ dans l'équation (9) devient $[14,16]$ :

$$
\begin{array}{rlrl}
G(\tau) & =\frac{\sin \beta \pi}{\pi \tau}\left[\frac{\tau}{\tau_{\mathrm{c}}-\tau}\right]^{\beta} & \text { pour } & 0<\tau \leqslant \tau_{\mathrm{c}} \\
& =0 & \text { pour } \tau>\tau_{\mathrm{c}}
\end{array}
$$

où $\beta$ donne l'amplitude de la dispersion (nulle pour $\beta$ égal à l'unité) et $\tau_{\mathrm{c}}$ est le temps de relaxation non nul pour lequel $G(\tau)$ est maximal. La figure 6 donne un

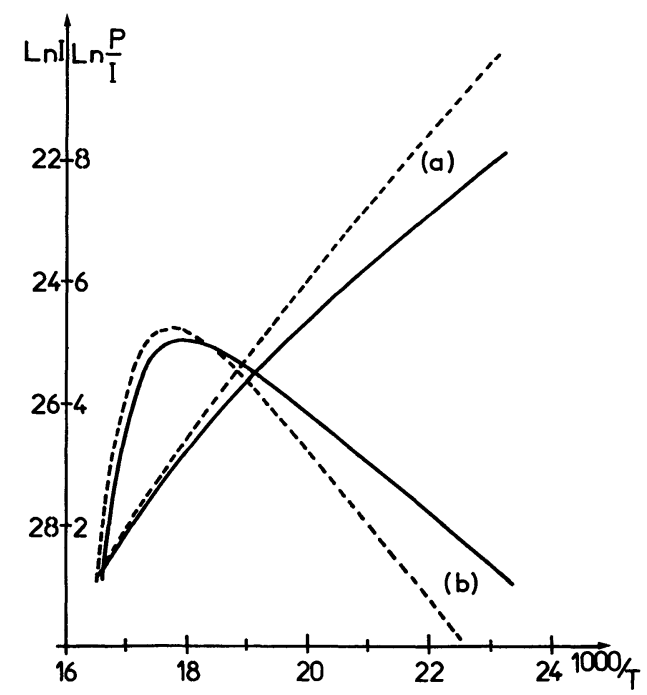

Fig. 6. - Courbe (a) : $\operatorname{Ln}(P / I)$ et courbe (b) $: \operatorname{Ln}(I)$ pour une distribution de Davidson et Cole : $\beta=0,7$ en traits interrompus, $\beta=0,5$ en traits continus, $\tau_{\mathrm{c}}=10^{-12} \mathrm{~s}, \quad E_{0}=0,15 \mathrm{eV}$ et $b=0,1 \mathrm{Ks}^{-1}$.

[Curve (a) : $\operatorname{Ln}(P / I)$ and curve (b) : $\operatorname{Ln}(I)$ for a Davidson and Cole distribution : $\beta=0.7$ in dashed lines, $\beta=0.5$ in continuous lines, $\tau_{\mathrm{c}}=10^{-12} \mathrm{~s}, E_{0}=0.15 \mathrm{eV}$ and $b=0.1 \mathrm{Ks}^{-1}$.] 


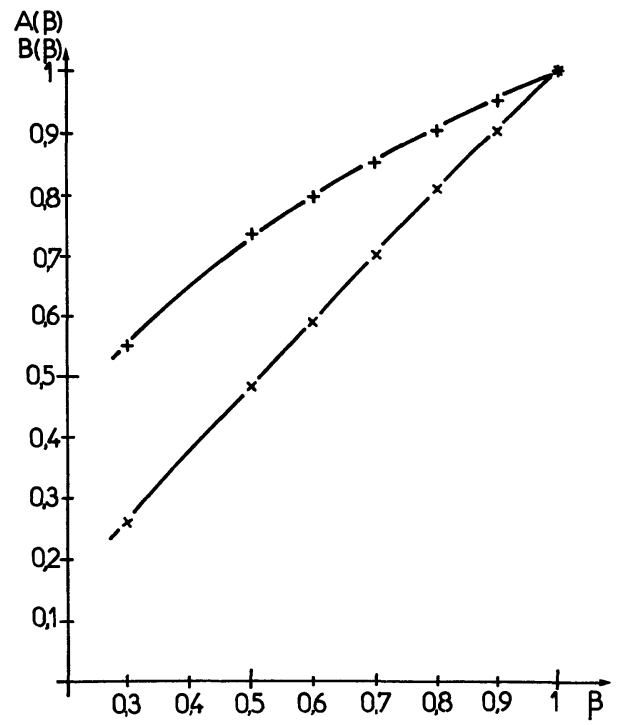

Fig. 7. $-+++: A(\beta)$ et $\times \times \times: B(\beta)$ pour une distribution de Davidson et Cole.

$[+++: A(\beta)$ and $\times \times \times: B(\beta)$ for a Davidson and Cole distribution.]

exemple des courbes obtenues pour une distribution de Davidson et Cole et la figure 7 donne graphiquement les coefficients $A(\beta)$ et $B(\beta)$ définis par les équations (6) et (7).

5. Exemples d'application de la méthode. - De nombreux corps polaires liquides, alcools, halogénures d'alcoyles ainsi que des polymères solides, présentent une distribution asymétrique des temps de relaxation diélectrique quand la température décroît et s'approche de celle de la transition vitreuse. Il est intéressant de voir dans quelle mesure ce résultat se modifie à plus basse température et c'est là que des mesures de TCD trouvent toute leur utilité.

La figure 8 reproduit les courbes du TCD : $I(T)$ et du rapport $P(T) / I(T)$ en fonction de $1 / T$ pour le 1-chloro-2-éthyl-hexane. Pour ce composé, nous avons déterminé $T_{\mathrm{g}}=122 \mathrm{~K}$ par extrapolation à $10^{13} \mathrm{P}$ de la courbe $\eta(T)$ que nous avons mesurée de 1 à $10^{8} \mathrm{P}$. Les TCD ont été obtenus après polarisation pendant $360 \mathrm{~s}$ vers $125 \mathrm{~K}$ sous champ électrique de $500 \mathrm{kVm}^{-1}$, suivi d'un refroidissement à $80 \mathrm{~K}$ en environ 15 min., suppression du champ électrique et enfin réchauffement de vitesse $0,05 \mathrm{Ks}^{-1}$ pour l'enregistrement du courant $I(T)$. Un pic de courant apparaît pour $T_{\mathrm{M}}=123,5 \mathrm{~K}$ (soit 8,12 en $10^{3} / T$ ). L'allure des flancs de ce pic est comparable à ceux du pic de la figure 6 , qui correspond à une distribution asymétrique des temps de relaxation. Nous savons $a$ priori qu'aucune distribution symétrique ne saurait convenir, les modules des pentes des deux flancs du pic étant trop différents.

La méthode exposée ci-dessus permet de déterminer directement les paramètres $\tau_{\mathrm{c}}$ et $E_{0}$ lorsque la loi de distribution est connue. Dans le cas des halogénures

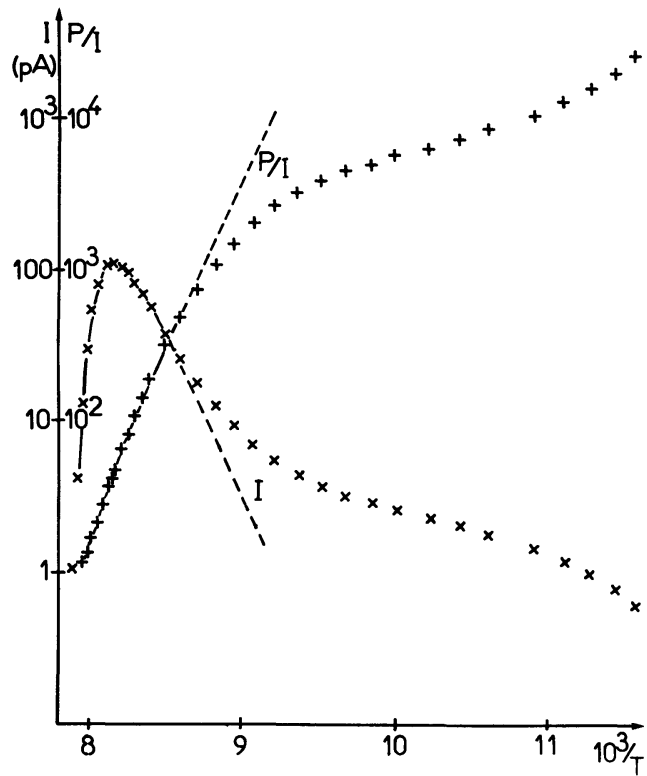

Fig. 8. - Pic expérimental de TCD du 1-chloro-2-éthyl-hexane pour une polarisation à $T_{\mathrm{p}}=125 \mathrm{~K}$ durant $360 \mathrm{~s}$ (voir les +++ et les $\times \times \times$ ). Le pic théorique (en tirets) pour $\tau_{\mathrm{c}}=10^{-35} \mathrm{~s}$, $E_{0}=0,9 \mathrm{eV}$ et $b=0,05 \mathrm{Ks}^{-1}$ diffère du pic expérimental si $1000 / T \geqslant 8,5$.

[Experimental DTC peak of 1-chloro-2-ethyl-hexane for a polarization at $T_{\mathrm{p}}=125 \mathrm{~K}$ during $360 \mathrm{~s}$ (see +++ and $\times \times \times$ ). The theoretical peak for $\tau_{:}=10^{-35} \mathrm{~s}, E_{0}=0.9 \mathrm{eV}$ and $b=0.05 \mathrm{Ks}^{-1}$ differs from the experimental one if $1000 / T \geqslant 8.5$ (in dashed lines).]

d'alcoyles liquides, donc pour le 1-chloro-2-ethylhexane, des mesures diélectriques conventionnelles ont permis d'observer une distribution asymétrique de $\tau$ dont le poids est donné par une équation de Davidson et Cole (éq. (14)). Le paramètre $\beta$ déterminant la largeur de cette distribution prend la valeur $\beta_{0}=0,5$ pour des températures qui s'approchent de celle de la transition vitreuse [17]. Dans ce cas, nous avons donc considéré cette valeur de $\beta$ comme représentant la largeur de la distribution à l'état vitreux.

Pour $\beta_{0}=0,5$ les courbes de la figure 7 donnent :

$$
\begin{aligned}
& A\left(\beta_{0}\right)=0,72 \\
& B\left(\beta_{0}\right)=0,48 .
\end{aligned}
$$

La valeur de $\operatorname{tg} \theta_{\mathrm{M}}$ pour $T_{\mathrm{M}}=123,5 \mathrm{~K}$ déduite des courbes expérimentales de la figure 8 est :

$$
\operatorname{tg} \theta_{M}=7,5 \times 1000=7500
$$

(le coefficient 1000 provient de la graduation en $1000 / T$ de l'échelle des abscisses).

$\mathrm{Si}$ l'on reporte ces valeurs ainsi que celle de $b=0,05 \mathrm{Ks}^{-1}$ dans les équations (4) et (5), on obtient :

$$
\begin{aligned}
E_{0} & =\frac{k \operatorname{tg} \theta_{\mathrm{M}}}{A\left(\beta_{0}\right)} \sim 0,9 \mathrm{eV} \\
\tau_{\mathrm{c}} & =\frac{T_{\mathrm{M}}^{2}}{B\left(\beta_{0}\right)} \frac{k}{b E_{0}} \exp \left(-\frac{E_{0}}{k T_{\mathrm{M}}}\right) \sim 10^{-35} \mathrm{~s} .
\end{aligned}
$$


Le pic théorique obtenu par simulation sur ordinateur avec ces valeurs numériques a été superposé aux courbes expérimentales sur la figure 8 . La concordance est excellente au voisinage de $T_{\mathrm{M}}=123,5 \mathrm{~K}$. Nous reviendrons sur l'écart qui existe à basse température.

Nous allons montrer comment cette même méthode s'applique lorsque la loi de distribution n'est pas connue. Pratiquement l'allure du pic de courant montre si la distribution est symétrique (modules de la pente des deux flancs du pic sensiblement égaux) ou asymétrique (modules des deux pentes très différents). Dans le cas de distributions symétriques, aussi bien une distribution gaussienne qu'une distribution de Cole et Cole permettent de rendre compte des courbes expérimentales. Pour les distributions asymétriques, comme nous l'avons déjà dit, nous avons choisi celle de Davidson et Cole, par continuité avec l'exploitation traditionnelle des résultats obtenus à l'état liquide. Dans le cas du pic pour $T_{\mathrm{M}}=123,5 \mathrm{~K}$ de la figure 8, pour obtenir une bonne coïncidence entre le flanc du pic expérimental pour $T \geqslant T_{\mathrm{M}}$ et le pic simulé, nous pouvons prendre n'importe lequel des couples de valeurs : $\beta=0,4$ et $E_{0}=1 \mathrm{eV}$, ou $\beta=0,5$ et $E_{0}=0,9 \mathrm{eV}$, ou $\beta=0,6$ et $E_{0}=0,8 \mathrm{eV}$. Par contre, pour $T \leqslant T_{\mathrm{M}}$, le meilleur accord est obtenu pour $\beta=0,5$ et $E_{0}=0,9 \mathrm{eV}$. En effet sur le flanc du pic à $T \leqslant T_{\mathrm{M}}$, la pente est proportionnelle à $E_{0}$ et sa variation avec $\beta$ est moins rapide que sur l'autre flanc, ce qui permet de déterminer aisément $E_{0}$. Une fois la distribution déterminée, le calcul de $\tau_{c}$ se fait comme précédemment.

Nous avons parlé plus haut de l'écart qui existe à basse température entre le pic simulé et le pic expérimental. Cet écart s'explique, du moins en partie, par l'existence d'un pic supplémentaire distribué, culminant vers $90 \mathrm{~K}$. De tels pics, appelés aussi distributions secondaires, ou pics $\beta$, sont observés dans la plupart des composés vitreux $[12,13]$. Leur fréquence est généralement 3 à 4 ordres de grandeur plus élevés que celle du pic principal, ou pic $\alpha$, à la même température, et ils ont été observés à l'aide des mesures diélectriques conventionnelles. Nous allons montrer comment on peut obtenir leur distribution à l'aide de la méthode des TCD.

Dans le cas du 1-chloro-2-ethyl-hexane, nous avons tout d'abord isolé ce pic en polarisant le produit à $90 \mathrm{~K}$, pendant $3600 \mathrm{~s}$, sous un champ de $500 \mathrm{kVm}^{-1}$. On montre aisément que, dans ces conditions, les processus correspondant au pic $\alpha$, celui qui culmine à $T_{\mathrm{M}}=123,5 \mathrm{~K}$, ne rentrent pas en jeu. Après quoi, l'échantillon a été refroidi à $77 \mathrm{~K}$. Il est probable qu'à cette température les mouvements moléculaires sont encore importants, si bien que la partie haute température du pic $\beta$ a pu être observée lors du réchauffement à partir de $77 \mathrm{~K}$ avec une vitesse $b=0,025 \mathrm{Ks}^{-1}$ (Fig. 9). Notons que sur la figure 8, le pic vers $90 \mathrm{~K}$ est aussi tronqué pour la même raison. Ce pic correspond à une distribution symé-

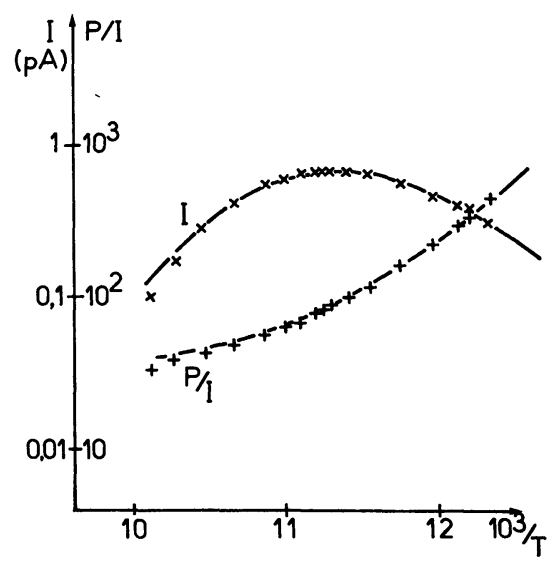

Fig. 9. - Pic expérimental de TCD du 1-chloro-2-éthyl-hexane pour une polarisation à $T_{\mathrm{p}}=90 \mathrm{~K}$ durant $3600 \mathrm{~s}$ (voir les +++ et les $\times \times \times$ ). Le pic théorique (en tirets) pour $\tau_{c}=10^{-10} \mathrm{~s}$, $E_{0}=0,20 \mathrm{eV}$ et $b=0,025 \mathrm{Ks}^{-1}$ se superpose au pic expérimental.

[Experimental DTC peak of 1-chloro-2-ethyl-hexane for a polarization at $T_{\mathrm{p}}=90 \mathrm{~K}$ during $3600 \mathrm{~s}$ (see +++ and $\left.\times \times \times\right)$. The theoretical peak (in dashed lines) for $\tau_{\mathrm{c}}=10^{-10} \mathrm{~s}, E_{0}=0.20 \mathrm{eV}$ and $b=0.025 \mathrm{Ks}^{-1}$ intermingles with the experimental peak.]

trique de $\tau$ puisque ses deux flancs ont les modules de leurs pentes comparables. Mais dans ce cas de polarisation incomplète les relations (4) et (5) ne s'appliquent plus.

Pour déterminer pratiquement les paramètres $E_{0}$, $\tau_{\mathrm{c}}$ et $\delta$ pour une distribution gaussienne (éq. (7), (7bis) et (10)), il faut donner des valeurs initiales à ces paramètres, puis les modifier légèrement pour améliorer la superposition des pics simulés avec le pic expérimental. Pour calculer des valeurs initiales nous utiliserons les relations (4) et (5) qui, bien qu'approchées, donnent un ordre de grandeur pour $E_{0}$ et $\tau_{\mathrm{c}}$. En prenant $\delta \sim 0,30$ pour paramètre de la distribution (éq. (10)), nous trouvons à partir des courbes de la figure 3 :

$$
\begin{aligned}
& A(\delta)=0,48 \\
& B(\delta)=0,28
\end{aligned}
$$

d'où, en reportant dans les équations (4) et (5) :

$$
\begin{aligned}
E_{0} & \sim 0,20 \mathrm{eV} \\
\tau_{\mathrm{c}} & \sim 10^{-9} \mathrm{~s} .
\end{aligned}
$$

Après avoir fait varier $\delta, E_{0}$ et $\tau_{c}$, nous trouvons finalement les valeurs suivantes pour le pic simulé superposable au pic expérimental :

$$
\delta=0,25 \quad E_{0}=0,20 \mathrm{eV} \quad \tau_{\mathrm{c}}=10^{-10} \mathrm{~s} .
$$

Notons qu'un pic de TCD simulé pour une distribution de Cole et Cole (éq. (11)) permet une aussi bonne superposition avec le pic expérimental pour :

$$
\alpha=0,50 \quad E_{0}=0,20 \mathrm{eV} \quad \tau_{\mathrm{c}}=10^{-10} \mathrm{~s} .
$$

Ce résultat illustre l'équivalence empirique des diverses distributions gaussiennes. Il faudrait des mesures de 
courant significatives jusqu'à $I\left(T_{\mathrm{M}}\right) / 1000$ pour pouvoir discriminer entre les différents types de ces distributions.

6. Discussion. - Afin de tester la validité de cette méthode d'exploitation quantitative des TCD, nous avons cherché à comparer les résultats décrits plus haut avec des résultats de mesures diélectriques conventionnelles dans le domaine liquide du même composé. Nous avons donc effectué de telles mesures de $126 \mathrm{~K}$ à $160 \mathrm{~K}$ dans une gamme de fréquence allant de $10^{-3} \mathrm{~Hz}$ à $3 \times 10^{6} \mathrm{~Hz}$. (De $30 \mathrm{~Hz}$ à $3 \times 10^{6} \mathrm{~Hz}$, nous avons utilisé deux ponts de Schering General Radio $716 \mathrm{C}$ et $716 \mathrm{CS}$; en ce qui concerne les très basses fréquences, nous nous sommes servis d'un montage comportant un générateur TBF Hewlett-Packard modèle 203A et un amplificateur-électromètre Keithley modèle $300 \mathrm{~A}$ ou $610 \mathrm{C}$, alternativement).

La figure 10 montre la variation en fonction de la température du temps de relaxation $\tau_{\mathrm{M}, \mathrm{D}}$ au maximum de l'absorption diélectrique. Comme le paramètre $\beta$ de la distribution de Davidson et Cole varie de 0,5 (à $126 \mathrm{~K}$ ) à $\sim 0,7$ (à $160 \mathrm{~K}$ ), nous avons vérifié que les courbes $\operatorname{Ln} \tau_{\mathrm{M}, \mathrm{D}}=\varphi(1 / T)$ et $\operatorname{Ln} \tau_{\mathrm{C}, \mathrm{D}}=\varphi(1 / T)$ ( $\tau_{C, D}$ fréquence caractéristique de la distribution, voir éq. (14)) sont pratiquement parallèles ; autrement dit, la variation de la pente de ces graphes, c'est-àdire de l'énergie d'activation apparente suivant l'équation (1), est pratiquement insensible à la façon dont on choisit les points homologues de la distribution pour représenter la variation du temps de relaxation en fonction de la température.

Par ailleurs, on peut faire varier la température $T_{\mathrm{M}}$ au maximum des courbes $\operatorname{Ln} I=\varphi(1 / T)$ de TCD

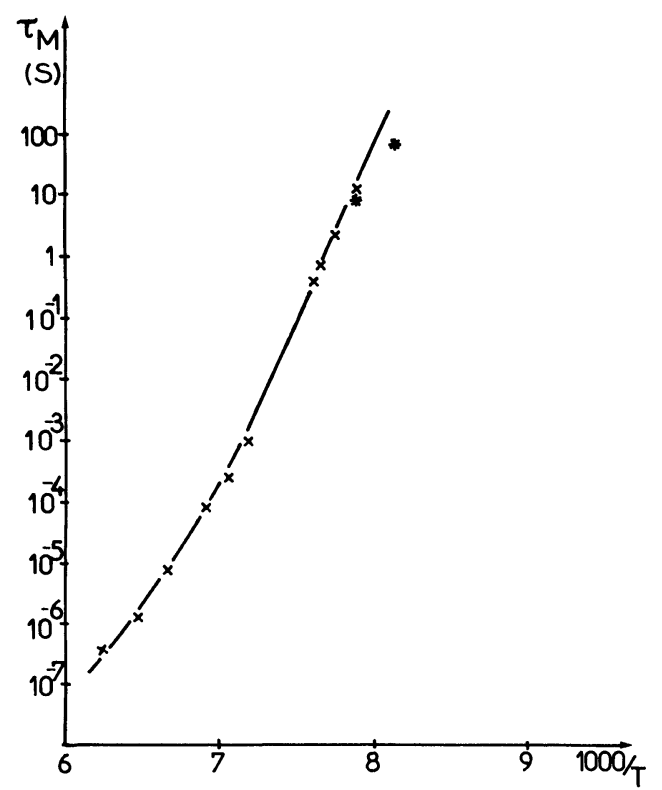

Fig. 10. $-\times \times \times: \tau_{M, D}$ et ${ }^{* * *}: \tau_{M . T C D}$ pour le 1-chloro-2-éthylhexane.

$\left[\times \times \times: \tau_{M . D}\right.$ and $* * *: \tau_{M . D T C}$ for 1-chloro-2-ethyl-hexane.] en modifiant la vitesse de réchauffement, $b$. Ainsi, en effectuant une mesure de TCD avec $b=0,15 \mathrm{Ks}^{-1}$, nous avons obtenu $T_{M}=126,6 \mathrm{~K}$, qui se trouve dans le domaine où nous avons réalisé les mesures diélectriques. Le temps de relaxation $\tau_{\mathrm{M} \text {,TCD }}$ en ce point est donné par (éqs. (1) et (5)) :

$$
\tau_{\mathrm{M}, \mathrm{TCD}}=\frac{T_{\mathrm{M}}^{2}}{B(\beta)} \frac{k}{b E_{0}} \text {. }
$$

Les valeurs de $\tau_{\mathrm{M}, \mathrm{D}}$ et $\tau_{\mathrm{M}, \mathrm{TCD}}$ obtenues pour une même température ne sont en principe pas rigoureusement identiques, car $\tau_{M, D}$ résulte d'une expérience isotherme, ce qui n'est pas le cas pour $\tau_{\mathrm{M}, \mathrm{TCD}}$. Mais la différence est négligeable comme Le Montagner [18] l'a montré par le calcul, du moins pour les vitesses de réchauffement utilisées ici.

Ainsi donc, comparant les résultats obtenus à $126,6 \mathrm{~K}$ pour les deux méthodes expérimentales, nous obtenons :

$$
\begin{aligned}
& E_{0}=1,3 \mathrm{eV} \tau_{\mathrm{M}, \mathrm{D}}=12,7 \mathrm{~s} \text { mesures diélectriques } \\
& E_{0}=1,2 \mathrm{eV} \quad \tau_{\mathrm{M}, \mathrm{TCD}}=7,9 \mathrm{~s} \mathrm{TCD} .
\end{aligned}
$$

Nous ne pensons pas que l'écart sur les valeurs de $E_{0}$ soit significatif, car $E_{0}$ (diélectrique) est déduit d'une mesure de pente de tangente, ce qui n'est jamais très précis. D'autre part, dans ce domaine expérimental proche de $T_{\mathrm{g}}$ où il suffit d'une élévation de température d'environ $0,8 \mathrm{~K}$ pour que $\tau_{M}$ double, l'écart que nous observons sur les valeurs de $\tau_{M, D}$ et $\tau_{M, T C D}$ peut s'expliquer par une différence de $0,5 \mathrm{~K}$. Etant donné que nous nous référons ici à des mesures de températures qui proviennent de deux montages expérimentaux différents et que, dans l'un de ces montages, les TCD, la température varie continuement, cet écart d'environ $0,5 \mathrm{~K}$ se situe certainement à l'intérieur de l'incertitude expérimentale. Dans ces conditions on peut dire que l'accord entre les valeurs de $\tau_{\mathrm{M}, \mathrm{D}}$ et $\tau_{\mathrm{M}, \mathrm{TCD}}$ citées plus haut est excellent.

Il nous faut maintenant comprendre pourquoi l'expérience de TCD décrite précédemment pour une vitesse de réchauffement plus faible $\left(b=0,05 \mathrm{Ks}^{-1}\right)$ donne une énergie d'activation beaucoup plus faible, soit :

$$
E_{0}=0,9 \mathrm{eV} \text { à } 123,5 \mathrm{~K} .
$$

Notons que l'échantillon a été polarisé $3 \mathrm{~min}$. à $125 \mathrm{~K}$, température à laquelle $\tau_{\mathrm{M}}$ est de l'ordre de $30 \mathrm{~s}$. On peut donc considérer que la polarisation a été complète.

Par contre, nous n'avons pas tenu compte, dans l'exploitation des résultats, de la polarisation supplémentaire qui se produit au cours du refroidissement du fait de l'augmentation de la permittivité liée, entre autres, à l'augmentation de la densité. Un tel phénomène ne serait évidemment sensible que pour les temps de relaxation les plus courts, c'est-à-dire pour la partie basse température de la courbe de 
TCD. Cela produirait, pour $T<T_{\mathrm{M}}$, un étalement de la courbe de $\operatorname{Ln} I=\varphi(1 / T)$, c'est-à-dire une diminution apparente à la fois de $\beta$ et de $E_{0}$. Or, ceci n'est pas compatible avec les valeurs des couples $\beta, E_{0}$ qui conviennent pour le domaine $T>T_{\mathrm{M}}$ (couples dans lesquels $\beta$ et $E_{0}$ varient en sens inverse, voir ci-dessus). Il en résulte que cet effet ne peut sûrement pas expliquer la diminution observée pour $E_{0}$.

Il nous faut donc admettre que cette diminution de $E_{0}$ aux alentours de $T_{\mathrm{g}}$ est un phénomène réel. Remarquons tout d'abord que $E_{0}$ est évalué soit à partir de la pente de la courbe $\tau_{\mathrm{M}, \mathrm{D}}=\varphi(1 / T)$, soit à partir de l'ajustement d'une courbe calculée et d'une courbe de TCD expérimentale. De toute façon, il est évident qu'une valeur de $E_{0}$ n'est valable que dans un intervalle restreint de température. Dans le cas présent, nous avons :

$$
\begin{array}{lll}
126-130 \mathrm{~K} & E_{0}=1,3 \mathrm{eV} & \text { (diélectrique) } \\
120-128 \mathrm{~K} & E_{0}=1,2 \mathrm{eV} & \text { (TCD) } \\
116-125 \mathrm{~K} & E_{0}=0,9 \mathrm{eV} & \text { (TCD) }
\end{array}
$$

Ainsi, nos résultats révèlent que la courbe

$$
\operatorname{Ln} \tau_{\mathrm{M}}=\varphi(1 / T)
$$

présente un point d'inflexion dans la région de la transition vitreuse (Fig. 9). Tammann avait déjà observé une inflexion analogue dans la courbe qui représente, en fonction de la température, la dureté de surface de composés amorphes telle qu'elle est mesurée par le degré de pénétration d'une bille [19]. Une observation du même ordre a été faite par Seki et ses collaborateurs [20] qui ont juxtaposé les résultats de relaxation diélectrique et de relaxation enthalpique, obtenus respectivement de part et d'autre de la transition vitreuse du cyclohexanol à l'état de cristal vitreux.

On peut donc penser que, à l'instar de la viscosité statique, $\eta$, dont l'évolution autour de $T_{\mathrm{g}}$ a été décrite par Prod'homme [21], le temps de relaxation $\tau_{\mathrm{M}}$ au maximum de l'absorption du domaine $\alpha$ ne tend pas vers l'infini à basse température, contrairement aux prévisions de certains travaux théoriques [22]. On est alors en droit de se demander si la proportionnalité entre $\tau_{\max }$ et $\eta$ décrite par Denney [3] pour les liquides surfondus ne se poursuit pas dans le domaine d'existence du verre; par extension du travail de Prod'homme sur la viscosité [21], on pourrait alors fixer la valeur limite de $\tau_{\max }$ vers $10^{7}-10^{10} \mathrm{~s}$.

7. Conclusions. - Dans ce travail nous avons présenté une méthode numérique d'exploitation des expériences de TCD qui généralise les méthodes proposées par ailleurs $[5,23,24,25]$.

Le but $a$ priori de cette étude était de décrire les propriétés diélectriques d'une matrice vitreuse dans le souci de les comparer à celles du liquide surfondu dont elle dérive. Nous avons donc choisi de représenter ces propriétés par des distributions de temps de relaxation habituelles dans la description des liquides, telles que la distribution symétrique de Cole et Cole ou la distribution asymétrique de Davidson et Cole. Nous avons admis que les fonctions de distribution utilisées restaient inchangées dans un intervalle de température d'une dizaine de degrés, la variation en fonction de la température étant gouvernée par une loi de type Arrhénius où l'énergie d'activation apparente $E_{0}$ reste constante dans le même domaine de température. Tout en étant conscients du caractère arbitraire de ces hypothèses, lesquelles n'apportent aucun élément de compréhension concernant l'origine physique des divers processus de relaxation, il nous a semblé indispensable d'obtenir une description empirique quantitative fidèle des phénomènes de relaxation diélectrique dans une matrice vitreuse, dans un domaine de fréquence où les mesures conventionnelles deviennent impuissantes ; cette description pourra servir de guide à toute modélisation ultérieure.

Cette méthode permet en principe de prendre en compte toute structure de fonction de distribution, même si la polarisation de l'échantillon n'est que partielle.

Pour cette étude, nous avons choisi un halogénure d'alkyle, chimiquement stable, dont nous savions par expérience qu'il ne subissait pas d'autre transformation que la transition vitreuse dans le domaine de température exploré. Nous avons donc pu lui faire subir toute une gamme de vitesses de réchauffement sans qu'une cristallisation malencontreuse ne vienne perturber la mesure.

En ce qui concerne les résultats, nous avons montré que, dans le faible domaine de température où l'on peut comparer les mesures diélectriques conventionnelles et les TCD, l'accord est excellent, ce qui semble valider cette dernière méthode.

Nos résultats ont aussi montré que le domaine $\alpha$ de relaxation principale continue d'exister en présence de la phase vitreuse, du moins pendant une dizaine de degrés; dans l'exemple étudié ce domaine présente une distribution de Davidson et Cole avec un paramètre de distribution $\beta$ égal à 0,5 comme dans le liquide surfondu à basse température, indice de la continuité entre les deux phases. Toutefois, il semble que l'énergie d'activation apparente du pic $\alpha$ diminue autour de $T_{\mathrm{g}}$. L'existence inconnue jusqu'à maintenant de cette inflexion dans la courbe $\operatorname{Ln} \tau_{\mathrm{M}}=\varphi(1 / T)$ explique sans doute pourquoi certains auteurs ont pensé que la loi de type WLF [26] :

$$
\tau_{M}=\tau_{0} \exp \left(\frac{E_{0}}{T-T_{x}}\right)
$$

valable pour le liquide surfondu tendait vers une loi d'Arrhénius $\left(T_{\infty} \rightarrow 0\right)$ au-dessous de $T_{\mathrm{g}}$ [13]. De plus ceci nous amène à penser que, à l'exemple de la viscosité d'un verre [21], $\tau_{M}$ tend, à basse température, vers une limite qui serait vers $10^{7}-10^{10} \mathrm{~s}$. 
Nous avons aussi démontré, dans ce composé, l'existence d'un domaine de relaxation secondaire, $\beta$, à basse température. L'énergie d'activation correspondant à ce pic, soit $0,2 \mathrm{eV}$, répond à ce que l'on pouvait attendre par comparaison avec d'autres composés $[12,13]$. Nous avons pu décrire la distribution des temps de relaxation de ce domaine, ce qui, à notre connaissance, est la première détermination du genre.

Malheureusement, nous ne disposons pas de mesures micro-ondes dans le composé liquide; nous ne savons donc pas si ce domaine $\beta$ est, comme dans d'autres exemples $[12,13]$ la suite du domaine de relaxation type Debye qui existe dans le liquide à haute température.

Pour le moment, la méthode que nous proposons permet d'exploiter les courbes de TCD dans le domaine proche de la transition vitreuse et, à plus basse température, au niveau d'un éventuel domaine $\beta$. Nous pensons, dans un proche avenir, pouvoir combler la lacune existant entre ces deux intervalles de température. Mais surtout, il nous faudra déterminer l'influence de l'histoire thermique de la matrice vitreuse, avant et pendant la polarisation, pour pouvoir tenir compte de l'évolution naturelle du milieu vitreux [27].

\section{Bibliographie}

[1] BucCI, C. and Fieschi, R., Phys. Rev. Lett. 12 (1964) 16.

[2] BucCI, C., Fieschi, R. and GuIDI, G., Phys. Rev. 148 (1966) 816.

[3] Denney, D. J., J. Chem. Phys. 30 (1959) 159.

[4] Tamman, G. and Hesse, W., Z. Anorg. Chem. 156 (1926) 245.

[5] van Turnhout, J., Central Lab. TNO, Delft, comm. n ${ }^{\circ} 471$ (1972).

[6] ONG, P. H. and van Turnhout, J., J. Electrochem. Soc. 119 (1972) C223.

[7] Schwarzl, F. and Staverman, A. J., J. Appl. Phys. 23 (1952) 838.

[8] Hino, T., J. Appl. Phys. 46 (1975) 1956.

[9] Hino, T. and Kitamura, Y., Elect. Eng. Japan 95 (1975) 24.

[10] Chatain, D., Lacabanne, C. and Monpagens, J. C., Makromol. Chem. 178 (1977) 583.

[11] Monpagens, J. C., Chatain, D. G., Lacabanne, C. and Gautier, P. G., J. Polymer Sci. Polymer Phys. Ed. 15 (1977) 767.

[12] Johari, G. P. and Goldstein, M., J. Chem. Phys. 53 (1970) 2372.

[13] Johari, G. P., The Glass Transition and the Nature of the Glassy State, ed. Goldstein, M. and Simha, R. (Academy Sc. New York) 279 (1976) 117.
[14] Connor, T. M., Trans. Farad. Soc. 60 (1964) 1574.

[15] Cole, K. S. and Cole, R. H., J. Chem. Phys. 9 (1941) 341.

[16] Davidson, D. W., Can. J. Chem. 39 (1961) 571

[17] Berberian, J. G. and Cole, R. H., J. Amer. Chem. Soc. 90 (1968) 3100

[18] Le Montagner, S., J. Physique radium 21 (1960) 69.

[19] Tamman, G., " Der Glaszustand " Voss, Leipzig (1933) 56.

[20] Suga, H., Seki, S., Kubota, S., Yamaguchi, S., Yano, O. and Wada, Y., Mol. Cryst. Liq. Cryst. 18 (1972) 345.

[21] Prod'homme, M. C., "Contribution à l'étude de la viscosité des verres", ed. Revue d'optique théorique et instrumentale, Paris (1960).

[22] Brereton, M. G. and Davies, G. R., Polymer 18 (1977) 764.

[23] Har moss, S., Physica 96B (1979) 129.

[24] Kryszewski, M., Zielinski, M. and SAPIeha, S., Polymer 17 (1976) 212.

[25] Fischer, P. and RöHL, P., J. Polymer Sci. Polymer Phys. Ed. 14 (1976) 543.

[26] Williams, M. L., Landel, R. F. and Ferry, J. D., J. Amer. Chem. Soc. 77 (1955) 3701 .

[27] Kovacs, A. J., Hutchinson, J. M. and AkLonis, J. J.. The Structure of non-crystalline Materials, ed. Gaskell, P. H. (Taylor and Francis, London) 1977, p. 153. 